\title{
Capacidades y actividades en el autocuidado del paciente con pie diabético
}

\author{
Balcázar-Ochoa Mayra ${ }^{1, a}$, Escate-Ruíz Yesseniaa ${ }^{2, a}$, Choque-Díaz Cristina ${ }^{a}$, Velásquez-Carranza Doris ${ }^{3, b}$
}

\section{RESUMEN}

Objetivo: determinar las capacidades y actividades del autocuidado en el paciente con pie diabético. Material y métodos: estudio cuantitativo, descriptivo de corte transversal, la población estuvo conformada por 60 pacientes que se encontraban hospitalizados por presentar pie diabético; se empleó como instrumento el cuestionario sobre capacidades y actividades de autocuidado del paciente con pie diabético, el cual fue elaborado por las investigadoras; la primera parte estuvo conformada por 13 ítems con preguntas abiertas, se obtuvo datos personales y antecedentes patológicos; la segunda parte, sobre capacidades de autocuidado, consideró cuatro dimensiones: sensación, destreza, habilidades aprendidas, y memoria y aprendizaje; conformado por cinco preguntas cada una; por último, se enfoca las actividades del autocuidado considerando cuatro dimensiones: asistencia al médico, dieta, cuidado del pie y ejercicio; conformado por cinco preguntas; para el procesamiento de datos se utilizó el programa estadístico SSPS versión 19. Resultados: $35 \%$ fueron mujeres y $65 \%$ varones, la edad promedio fue 65 años a más; $53,3 \%$ presentó instrucción secundaria completa; las capacidades y actividades fueron inadecuadas en un 68,3\%; las capacidades, según sus dimensiones, fueron inadecuadas: destreza en un 58\%; habilidades aprendidas en $61,7 \%$; memoria y aprendizaje $75 \%$, y sensación $60 \%$. Con relación a las actividades, las dimensiones fueron inadecuadas como asistencia al médico con $65 \%$, dieta $50 \%$, cuidado del pie 58,3\% y ejercicio 56,7\%. Conclusiones: las capacidades y actividades de autocuidado en un paciente con pie diabético son inadecuadas.

Palabras clave: autocuidado, pie diabético, cuidados de enfermería. Perú (Fuente DeCs BIREME).

\section{The capabilities and activities of self-care in patients with diabetic foot}

\begin{abstract}
Objetive: To determine the capabilities and activities of self-care in patients with diabetic foot. Material and Methods: Quantitative, descriptive cross-sectional study, the population consisted of 60 patients who were found hospitalized with diabetic foot; a «Questionnaire on skills and self-care activities of patient with diabetic foot», was used as an instrument which was developed by the researchers; the first part consisted of 13 items with open questions, personal data and history of other diseases; the second part consisted of self - care skilled patients as 4-dimensional sense, skill, learned skills, memory and learning; with 5 questions each; finally considered self-care activities such as 4-dimensional medical care, diet, foot care and exercise targets; consists of 5 questions; data processing for the statistical program SPSS version 19. Results: Were used: $35 \%$ were female and 39.65\% male, mean age was 65 years later, $53.3 \%$ have completed secondary education; capabilities and activities were inadequate in $68.3 \%$, according to size capabilities were inadequate as a skill by $58 \%, 61.7 \%$ learned skills, memory and learning $75 \%$ and $60 \%$ feeling. In relation to activities dimensions were inadequate and medical care $65 \%, 50 \%$ diet, foot care, $58.3 \%$ and $56 \%$ exercise. Conclusions. The skills and self-care activities in a patient with diabetic foot are inadequate.
\end{abstract}

Key words: Self-care, diabetic foot, nursing care. Peru (Source DeCs BIREME).

\footnotetext{
${ }^{1}$ Clínica San Vicente. Lima, Perú.
}

${ }^{2}$ Clínica Cayetano Heredia. Lima, Perú.

${ }^{3}$ Facultad de Enfermería. Universidad Peruana Cayetano Heredia. Lima Perú.

aLicenciada en Enfermería ; 'Magister en Enfermería. 


\section{INTRODUCCIÓN}

La diabetes mellitus (DM) es un problema de salud pública, pues es una de las enfermedades más frecuentes en la clínica humana. Según la Organización Mundial de la Salud (OMS), en el mundo hay 347 millones de personas con esta enfermedad (1); en el Perú afecta a casi 2 millones de personas, el $6-10 \%$ de la población sufre de diabetes mellitus, de este total el $12-15 \%$ padecen de pie diabético; casi $30 \%$ está hospitalizado y, de este porcentaje, un 25\% debe ser amputado (2). Según la Fundación Para la Diabetes, esta es una enfermedad crónica incurable (3). La DM se caracteriza por la elevada concentración de azúcar en sangre (hiperglucemia) producto de la descompensación en la producción de insulina o por un inadecuado uso de este por parte del organismo (4). Los síntomas que puede presentar la persona son diversos como: poliuria, polidipsia y polifagia (5). Esta enfermedad tiene complicaciones agudas y crónicas como la microangiopatía donde encontramos la neuropatía periférica que incluye pie diabético (6).

El pie diabético, según la Sociedad Española de Angiología y Cirugía Vascular es: «Una alteración clínica de base etiopatogénica neuropática e inducida por la hiperglucemia mantenida, en la que con o sin coexistencia de isquemia, y previo desencadenante traumático, produce lesión y/o ulceración del pie» (7). Esta afección repercute negativamente en la calidad de vida de los pacientes debido a los ingresos a establecimientos de salud con estadías hospitalarias prolongadas y amputaciones de los miembros inferiores por la progresión de las heridas; aproximadamente, un 50 a $70 \%$ de esos pacientes sufrirá amputación; por ello, se considera a esta enfermedad como problema de salud pública en todo el mundo, lo que se evidencia con altas tasas de morbilidad y mortalidad (8).

De acuerdo con la teoría de Dorothea Orem, la capacidad es la agencia de autocuidado, la cual lo define como un repertorio de habilidades y tipos de conocimientos de los individuos que tienen y usan para participar en una variada gama de intentos prácticos, esta se desarrolla en el curso de la vida diaria por medio de un proceso espontáneo de aprendizaje; es completada con la curiosidad intelectual, la instrucción y supervisión de otros, y con la experiencia de llevar a cabo las medidas de autocuidado. Pero las capacidades se ven afectadas por factores genéticos y constitucionales tanto como por la cultura, experiencia de la vida y el estado de salud (9). Por otro lado, las actividades de autocuidado consisten en las acciones deliberadas que las personas realizan para regular su funcionamiento y desarrollo en beneficio de su vida, salud y bienestar (10).

El desconocimiento de los dos conceptos anteriores influyen en el ingreso de los pacientes a los hospitales por presentar la complicación de pie diabético, ello se evidencia en el control inadecuado de glicemias, tabaquismo, sedentarismo y aquellos cuidados especiales que eviten esta complicación, como el no usar zapatos ajustados, el corte de uñas por personas especializadas, el uso de cremas humectantes, entre otros; por tanto, la enfermera tiene un importante rol en la educación y sensibilización de la importancia en el cumplimiento del tratamiento que requiere la participación activa del individuo en el cuidado de sí mismo, enfatizando así a una de las funciones de enfermería que es docencia.

La investigación tuvo como objetivo general determinar las capacidades y actividades del autocuidado en el paciente con pie diabético.

\section{MATERIAL Y MÉTODOS}

Estudio descriptivo de corte transversal realizado en pacientes con pie diabético hospitalizados en el servicio de Medicina del Hospital Nacional Alberto Sabogal Sologuren, en el periodo de enero - marzo de 2014. La población estuvo conformada por 60 pacientes con edad de 65 años a más y de ambos sexos, que cumplían con los criterios de inclusión, entre ellos: pacientes que presenten complicación de pie diabético según historia clínica en un periodo mínimo de 10 años, y con diabetes tipo 2 .

Para la recolección de datos se utilizó un instrumento elaborado por las investigadoras, basado en el cuestionario realizado por Backsheider en el año 1974 (11) denominado capacidades, actividades y necesidades del paciente diabético controlado en forma ambulatoria. El instrumento fue dividido en tres partes: la primera parte contiene datos personales como edad, sexo, grado de instrucción, lugar de procedencia, tiempo de enfermedad, registro de peso, talla, última glicemia, así como antecedentes familiares y presencia de otras enfermedades; en la segunda parte identifica las capacidades de autocuidado que presenta el paciente, consta de 20 ítems, 5 por cada dimensión que son: sensación, destreza, habilidades aprendidas, memoria y aprendizaje; la tercera parte identifica las actividades de autocuidado que presenta el paciente, contiene 20 ítems, 5 por cada dimensión que son: asistencia al médico, dieta, cuidado del pie y ejercicio.

Todos los ítems del instrumento son positivos y se califican con la escala de Lickert, que mantiene una escala 
ordinal de 1 a 5 (parte de lo negativo a lo positivo). El instrumento tiene una calificación global que corresponde al total de las capacidades y actividades, aplica la técnica de los promedios, y considera inadecuada de $0-112$ y adecuada $>$ de 112. Por otro lado, se clasificó la variable «capacidades» con un promedio de inadecuado de 0 a 60 puntos y adecuado > a 60; la variable «actividades» se consideró inadecuado con promedio de 0 a 52 y adecuado $>$ a 52 puntos.

El instrumento para su validación fue sometido a juicio de expertos evaluado por diez profesionales, donde se evaluó la concordancia entre ellos mediante la prueba binomial. Posteriormente, se realizó una prueba piloto con 30 pacientes con las mismas características de la población, se obtuvo la confiabilidad aceptable del instrumento utilizando el alfa de Cronbach.

El proyecto fue aprobado por el Comité Institucional de Ética de la Universidad Peruana Cayetano Heredia. Se consideró la confiabilidad de los datos y los principios bioéticos, también la firma del consentimiento informado de todos los participantes. Para el análisis de datos se utilizó el programa SPSS versión 19.

\section{RESULTADOS}

En la Tabla 1, la población estudiada fue de 60 pacientes hospitalizados en el Servicio de Medicina - área pie diabético, de los cuales $21(35,0 \%)$ fueron de sexo femenino y $39(65 \%)$ varones; el $63,3 \%$ de los pacientes tenía una edad de 65 años a más; y el 53,3\% mostraba grado de instrucción secundaria completa.

En la Tabla 2, El 68,3\% de los pacientes con pie diabético presentó nivel inadecuado de capacidades y actividades de autocuidado, mientras que el $31,7 \%$ presentó un nivel adecuado.
Tabla 1. Distribución de los pacientes hospitalizados del servicio de medicina - área pie diabético del Hospital Alberto Sabogal Sologuren según datos demográficos, enero - marzo 2014

\begin{tabular}{lrr}
\hline Datos demográficos & $(\mathbf{N}=60)$ & \multicolumn{1}{c}{$\%$} \\
\hline Edad & & \\
25 a 44 años & 1 & 1,7 \\
45 a 64 años & 21 & 35,0 \\
65 a más & 38 & 63,3 \\
Total & 60 & 100,0 \\
Sexo & & \\
Femenino & 21 & 35,0 \\
Masculino & 39 & 65,0 \\
Total & 60 & 100,0 \\
& & \\
G rado de instrucción & & 3,3 \\
Sin instrucción & 2 & 13,3 \\
Primaria & 8 & 53,3 \\
Secundaria & 32 & 23,3 \\
Sup. técnica & 14 & 6,7 \\
Sup. universitaria & 4 & 100,0 \\
Total & 60 &
\end{tabular}

Tabla 2. Distribución de los pacientes hospitalizados del Servicio de Medicina - área pie diabético del Hospital Alberto Sabogal Sologuren según capacidades y actividades de autocuidado de acuerdo al sexo, enero - marzo 2014

\begin{tabular}{|c|c|c|c|c|c|c|}
\hline \multirow{3}{*}{ Sexo } & \multicolumn{4}{|c|}{ Capacidades y actividades } & \multirow{2}{*}{\multicolumn{2}{|c|}{ Total }} \\
\hline & \multicolumn{2}{|c|}{ Inadecuado } & \multicolumn{2}{|c|}{ Ad ecuado } & & \\
\hline & $\mathrm{N}:{ }^{\circ}$ & $\%$ & N. ${ }^{\circ}$ & $\%$ & $\mathrm{~N}^{\circ}$ & $\%$ \\
\hline Femenino & 14 & 66,7 & 7 & 33,3 & 21 & 100 \\
\hline Masculino & 27 & 69,2 & 12 & 30,8 & 39 & 100 \\
\hline Total & 41 & 68,3 & 19 & 31,7 & 60 & 100 \\
\hline
\end{tabular}

Tabla 3. Distribución de los pacientes hospitalizados del Servicio de Medicina - área pie diabético del Hospital Alberto Sabogal Sologuren según dimensiones de capacidades de autocuidado, enero - marzo 2014

\begin{tabular}{|c|c|c|c|c|c|c|c|c|}
\hline \multirow[b]{2}{*}{ Nivel } & \multicolumn{2}{|c|}{ Destreza } & \multicolumn{2}{|c|}{$\begin{array}{l}\text { Habilidades } \\
\text { aprendidas }\end{array}$} & \multicolumn{2}{|c|}{$\begin{array}{l}\text { Memoria y } \\
\text { aprendizaje }\end{array}$} & \multicolumn{2}{|c|}{ Sensación } \\
\hline & N. ${ }^{\circ}$ & $\%$ & N. ${ }^{\circ}$ & $\%$ & N. ${ }^{\circ}$ & $\%$ & N. ${ }^{\circ}$ & $\%$ \\
\hline Inadecuado & 35 & 58,3 & 37 & 61,7 & 45 & 75,0 & 36 & 60,0 \\
\hline Adecuado & 25 & 41,7 & 23 & 38,3 & 15 & 25,0 & 24 & 40,0 \\
\hline Total & 60 & 100,0 & 60 & 100,0 & 60 & 100,0 & 60 & 100,0 \\
\hline
\end{tabular}


Tabla 4. Distribución de los pacientes hospitalizados del Servicio de Medicina - área pie diabético del Hospital Alberto Sabogal Sologuren según dimensiones de capacidades de autocuidado, enero - marzo 2014

\begin{tabular}{|c|c|c|c|c|c|c|c|c|}
\hline \multirow[b]{2}{*}{ Nivel } & \multicolumn{2}{|c|}{$\begin{array}{l}\text { Asistencia al } \\
\text { médico }\end{array}$} & \multicolumn{2}{|c|}{ Dieta } & \multicolumn{2}{|c|}{$\begin{array}{l}\text { Cuidado } \\
\text { del pie }\end{array}$} & \multicolumn{2}{|c|}{ Ejercicio } \\
\hline & N. & $\%$ & N. ${ }^{\circ}$ & $\%$ & $\mathrm{~N}{ }^{\circ}$ & $\%$ & N. ${ }^{\circ}$ & $\%$ \\
\hline Inadecuado & 39 & 65,0 & 30 & 50,0 & 35 & 58,3 & 34 & 56,7 \\
\hline Adecuado & 21 & 35,0 & 30 & 50,0 & 35 & 41,7 & 26 & 43,3 \\
\hline Total & 60 & 100,0 & 60 & 100,0 & 60 & 100,0 & 60 & 100,0 \\
\hline
\end{tabular}

En la Tabla 3, respecto al nivel de capacidades de autocuidado, los resultados mostraron que $75,0 \%$ de los pacientes presentaron un nivel inadecuado de esas capacidades. De la variable «capacidades» se analizaron cuatro dimensiones entre ellas: destreza, habilidades aprendidas, memoria y aprendizaje y sensación; evidenciándose que estas dimensiones poseen mayor porcentaje en aquellos pacientes con capacidades inadecuadas.

En la Tabla 4, en el nivel de actividades de autocuidado, el $58,3 \%$ de pacientes presentó un nivel inadecuado de actividades. Se estudiaron cuatro dimensiones de la variable «actividades de autocuidado»: asistencia al médico, dieta, cuidados del pie y ejercicio; donde se observó que los pacientes presentaron, en su mayoría, actividades inadecuadas correspondientes a cada dimensión, de la cual «asistencia al médico» presenta un mayor porcentaje con 65,0 $\%$ de actividades inadecuadas.

\section{DISCUSIÓN}

Respecto a las características sociodemográficas, se obtuvo que el $63,3 \%$ de los pacientes son de 65 años a más, el $65 \%$ de sexo masculino y el $53,3 \%$ con grado de instrucción secundaria completa.

En cuanto a las capacidades y actividades de autocuidado de los pacientes con pie diabético que ingresan al Servicio de Medicina, el 68,3\% presenta nivel inadecuado, resultados similares encontró Irasema R. quien en su estudio refiere que sus pacientes presentaron un $66,5 \%$ de capacidades de autocuidado regulares (12). En lo referente a la diabetes, es importante que los pacientes identifiquen las causas de las complicaciones y cuáles son estas, debido a que al conocerlas están en capacidad de realizar su autocuidado y así favorecer la efectividad de su tratamiento y/o controlarlo, evitando la presencia de posibles complicaciones que pueden agravar su estado.
Con relación a las capacidades de autocuidado, se observa que la dimensión «memoria y aprendizaje» presenta un resultado del 75,0\%, que es el mayor porcentaje frente a las otras tres dimensiones, ubicándose en el nivel inadecuado, pues da a conocer que el paciente tiene un déficit en el conocimiento sobre su enfermedad e interés sobre ella. En el estudio de Pérez A., se evidenció que la intervención educativa contribuye a elevar el nivel de conocimientos de los pacientes sobre su enfermedad, mejora la adhesión al tratamiento y el control metabólico; además, refiere que es posible, mediante un adecuado tratamiento, retrasar o incluso prevenir las complicaciones a largo plazo de la enfermedad, donde uno de los pilares principales es la educación sobre diabetes (13).

De acuerdo a las actividades de autocuidado se puede observar que la actividad que realizan más los pacientes es el ejercicio $(56,7 \%)$, resultados similares encontró Hidalgo, pues reconoce que $69,0 \%$ de los pacientes realiza ejercicios, de ellos $89,9 \%$ realiza caminata (14). Del mismo modo, Salcedo A. encontró que la actividad que realizaron más sus pacientes fue caminata con un 48,0\% (15). Uno de los elementos clave en el tratamiento de la diabetes es el ejercicio, pues tiene muchos efectos benéficos y contribuye a disminuir la glucosa, mejora la oxigenación, aumenta el gasto energético, mejora la presión arterial y el perfil lipídico, también controla el peso; además, el ejercicio es más accesible a los pacientes y es recomendado realizarlo durante 20 - 30 minutos al día (16).

Por otro lado, dentro de esta dimensión se tomó en cuenta la higiene, donde se observa un mayor porcentaje de nivel inadecuado, a diferencia de lo hallado por Peñalosa donde los participantes tenían muy buenas capacidades de autocuidado en cuanto a los hábitos de higiene. El desarrollo y la implementación de hábitos higiénicos son necesarios para el paciente diabético, dado que este es proclive a desarrollar neuropatía de las fibras nerviosas sensitivas y motoras, produciendo diferentes manifestaciones en 
los miembros inferiores como: disminución de la sensibilidad profunda y superficial; piel seca y agrietada que produce fisuras que son el inicio de una lesión o la puerta de entrada a la infección. Por tal razón, la higiene en el paciente diabético es primordial para evitar lesiones que podrían traer complicaciones (17).

En la dimensión «cuidado del pie» el 58,3\% presentó un nivel inadecuado; así también, Méndez $\mathrm{V}$. encontró que al realizar exploración de los pies un 50,0\% de las usuarias mostraron lesiones, es decir, callosidades, micosis y mal olor. Esto se debe a su condición precaria y al uso de calzado de tela, plástico y huaraches de piel, siendo este último de uso tradicional (18).

Por último, se puede observar que la dimensión «dieta» es la que presenta un mayor puntaje de actividades adecuadas con un $50,0 \%$ frente a las otras tres dimensiones, resultados diferentes se encontraron en el estudio de Cabrera M. con respecto a la dieta, ya que del total de usuarios el 51,4\% no conoce cuál es la importancia de la dieta balanceada (19), ingerir de 4 a 6 comidas por día y evitar los azúcares de absorción rápida, controlar el colesterol, lípidos (grasas o aceites), consumir en cantidades limitadas (20). Así mismo; la actividad que presenta un mayor porcentaje de nivel inadecuado es "asistencia al médico» con un valor de $65,0 \%$ a diferencia de las otras tres dimensiones. Resultados similares encontró Gallardo, quien refiere que le llamó la atención que algunos pacientes que encuestó aún mantenían el criterio de aplicarse tratamientos por sí solos en el hogar, lo que constituye un hábito desfavorable dentro su autocuidado que repercute negativamente en la evolución de su enfermedad (21).

\section{REFERENCIAS BIBLIOGRÁFICAS}

1. Organización Mundial de la Salud [Internet]. Naciones Unidas (citado 25 Sep 2011). Disponible desde: http:/ /www.who.int/mediacentre/factsheets/fs312/es/ index.html visitado el: 16 de Julio del 2012.

2. Ministerio de Salud [Internet]. Lima, Perú: Ministerio de Salud (citado 17 jul 2012). Disponible en: http:// www.minsa.gob.pe/index.asp?Op=2

3. Fundación para la diabetes [Internet]. Madrid, España 2008: Fundación para la diabetes.com (citado $16 \mathrm{Jul}$ 2012). Disponible en: http://www.fundaci ondiabetes.org/frames.asp?Url=/quienes/cont 03.htm\& frame=/top01.htm

4. National Diabetes Information Clearinghouse (NDIC). Neuropatía Diabética. Estados Unidos: Publicación N. ${ }^{\circ} 11 ; 29$ Junio 2012
5. Tébar F, Escobar F. La diabetes mellitus en la práctica clínica. Madrid, España: Panamericana; 2009

6. Smeltzer S, Bare B. Enfermería medicoquirúrgica. 10. ${ }^{\circ}$ ed. México: Mc Graw Hill; 2007

7. Rol del podólogo ante el pie diabético (Internet). 2010 (citado 1 Jun 2012). Disponible en: http://www.ulce ras.net/publicaciones/ROLDELPODO LOGOANTEELPIEDIABETICO.pdf

8. Gallardo U, Zangronis L, Hernández L. Perfil epidemiológico del pie diabético. La Habana, Cuba: Instituto Nacional de Angiología y Cirugía Cardiovascular [citado 16 Jul 2012]. Disponible en: http://bvs.sld.cu/revistas/ang/vol5_1_04/ ang16104.htm

9. Orem DE. Normas prácticas en enfermería: editorial ciencias médicas; 1980 . pp. 112 - 113 .

10. Taylor S, Marriner A, Rayle M. La teoría de enfermería del déficit de autocuidado. Modelos y teorías de enfermería. $6 .^{\circ}$ ed. Madrid, España: Elsevier; 2007. Pp. 189 - 202.

11. Backsheider JE. Self-Care Requirements, Self-Care Capabilities, and Nursing Systems In The Diabetic Nurse Management Clinic [Internet]. 1974, Dic. Am J Public Health (citado 2 Jun 2014). 64(12): 11381146. Disponible en: http://www.ncbi.nlm.nih.gov/ pmc/articles/PMC1775710/?page $=4$

12. Irasema R, Manoel A, Tatiane A, Maria Z. Autocuidado de personas con diabetes mellitus atendidas en un servicio de urgencia en México [Internet]. 2010, Nov. - Dic. (citado 30 Ago 2012); 18(6). Disponible en: http://www.google.com.pe/url? $\mathrm{Sa}=\mathrm{t} \& \mathrm{rct}=\mathrm{j} \& \mathrm{q}=\&$ esrc $=\mathrm{s} \&$ frm $=1 \&$ source $=$ web $\& \mathrm{~cd}=1 \&$ ved $=0$ cccqfjaa $\&$ url $=$ http $\% 3 \mathrm{~A} \% 2 \mathrm{~F} \% 2 \mathrm{Fwww}$. scielo.br $\% 2 \mathrm{Fpdf} \% 2 \mathrm{Frlae} \% 2 \mathrm{Fv} 18 \mathrm{n} 6 \% 2 \mathrm{Fes} 21 . \mathrm{pdf} \&$ ei=iphzu_T_GK238gHi0oGYCA\&usg=afqjenehy 5 rda9rkb2xspxmlfn g8dqwg\&bvm=bv.65397613,d.awc

13. Jordán Severo T, Oramas González R, González Cárdenas L. Rev. Cubana. Evaluación del efecto de una intervención educativa en los pacientes diabéticos de cuatro consultorios: Med Gen Integr. 2007 abril junio; 23 (2). Disponible en: http://scielo.sld.cu/scielo. php?Pid $=$ S086421252007000200007\&script=sci_arttext.

14. Hidalgo C, Eva V. Medidas de autocuidado que realizan los pacientes diabéticos - Factores socioculturales que favorecen o limitan su cumplimiento en los pacientes que asisten al Programa de Diabetes del Hospital Nacional Dos de Mayo Octubre-diciembre 2005 [tesis]. Lima, Perú: Hospital Nacional Dos de Mayo; 2005.

15. Salcedo A, Alba J, García E. Dominio cultural del autocuidado en diabéticos tipo 2 con y sin control glucémico en México [Internet]. 2008, abr. (citado el 
28 de Sep de 2012); 42(2). Disponible en: http:// dx.doi.org/10.1590/S0034-89102008000200010.

16. Fundación Española del Corazón. Diabetes y ejercicio [Internet]. España: FEC; 2014 (citado 23 Mar 2014). Disponible en: http://www.fundaciondelcorazon.com/ ejercicio/para-enfermos/980-diabetes-y-ejercicio.html

17. Herrera A. Personas con diabetes mellitus tipo 2 y su capacidad de agencia de autocuidado [Internet]. Cartagena, Colombia. 2012, Jul. (citado 2 Jun 2014): XXX (2): pp. 39-46. Disponible en: http:// www.revistas.unal.edu.co/index.php /avenferm/ article/view/36079/37474

18. Méndez V, Morales M, Pérez M. Autocuidado de las adultas mayores con diabetes mellitus inscritas en el programa de enfermedades crónicas en Temoaya en México. Ciencia enferm. [Internet]. 2010, Dic. (citado 16 Jul 2012); 16(3): pp. 103 - 109. Disponible en: http://www.scielo.cl/scielo.php?Script=sci_art text\&pid=S0717-95532010000300011\&lng=es. Doi:10.4067/S0717-95532010000300011.

19. Cabrera R, Motta I, Rodríguez C, Velásquez D. Nivel de conocimiento sobre autocuidado en la prevención de complicaciones diabéticas en usuarios del policlínico Chincha. Revista Enfermería Herediana. [Internet]. 2010. [citado 8 Jun 2012]; 3(1): pp. 29-36. Disponible en: http://www.upch.edu.pe/faenf/revfae/ Autocuidado\%203_4.pdf.

20. Sociedad andaluza de nutrición clínica y dietética [Internet]. Andalucía, España; copyright [citado 6 mar 2013]. Disponible en: http://sancyd.es/comedores/ discapacitados/menu.dieta.diabetes.php

21. F.J. Tébar, F. Escobar. La diabetes mellitus en la práctica clínica. Madrid, España; Panamericana: 2009

\section{Correspondencia}

Yessenia Escate Ruíz

Universidad Peruana Cayetano Heredia

Dirección: Av. Honorio Delgado 430, Urb. Ingeniería, Lima - 31

Correo electrónico: yessenia.escate@upch.pe

Forma de citar este artículo: Balcázar-Ochoa M, Escate-Ruíz Y, Choque-Díaz C, Velásquez-Carranza D. Capacidades y actividades en el autocuidado del paciente con pie diabético. Rev enferm Herediana. 2014;7(2):63-68. 